\title{
Article
}

\section{BrLETM2 Protein Modulates Anthocyanin Accumulation by Promoting ROS Production in Turnip (Brassica rapa subsp. rapa)}

\author{
Hyon Dok Song ${ }^{1,2,3,+}$, Jianfei Yang 1,2, ${ }^{+}$, Nam Hyok Mun ${ }^{1,2}$, Bowei Chen ${ }^{1,2}$, Yunzhu Chen ${ }^{1,2}$, Pyol Kim ${ }^{1,2}$, \\ Saneyuki Kawabata ${ }^{4}$, Yuhua Li ${ }^{1,2, *}$ and Yu Wang ${ }^{1,2, *(D)}$ \\ 1 Key Laboratory of Saline-alkali Vegetation Ecology Restoration (Northeast Forestry University), \\ Ministry of Education, Harbin 150040, China; shd@nefu.edu.cn (H.D.S.); yjf19910304@126.com (J.Y.); \\ mnh@nefu.edu.cn (N.H.M.); chenbw@nefu.edu.cn (B.C.); cyzcarol@foxmail.com (Y.C.); \\ kimpyol1119@nefu.edu.cn (P.K.) \\ 2 College of Life Science, Northeast Forestry University, Harbin 150040, China \\ 3 Korean Horticultural Association, Pyongyang 999093, Democratic People's Republic of Korea \\ 4 Institute for Sustainable Agroecosystem Services, Graduate School of Agricultural and Life Sciences, \\ The University of Tokyo, Midoricho, Nishitokyo, Tokyo 188-0002, Japan; skawabata@g.ecc.u-tokyo.ac.jp \\ * Correspondence: lyhshen@126.com (Y.L.); wangyu@nefu.edu.cn (Y.W.); \\ Tel.: +86-451-82191733 (Y.L.); +86-451-82191783 (Y.W.) \\ + These authors contributed equally to this work.
}

check for updates

Citation: Song, H.D.; Yang, J.; Mun, N.H.; Chen, B.; Chen, Y.; Kim, P.;

Kawabata, S.; Li, Y.; Wang, Y. BrLETM2 Protein Modulates Anthocyanin Accumulation by Promoting ROS Production in Turnip (Brassica rapa subsp. rapa). Int. J. Mol. Sci. 2021, 22, 3538. https://doi.org/ $10.3390 /$ ijms 22073538

Academic Editor: Joana Oliveira

Received: 15 February 2021

Accepted: 25 March 2021

Published: 29 March 2021

Publisher's Note: MDPI stays neutral with regard to jurisdictional claims in published maps and institutional affiliations.

Copyright: (C) 2021 by the authors Licensee MDPI, Basel, Switzerland. This article is an open access article distributed under the terms and conditions of the Creative Commons Attribution (CC BY) license (https:// creativecommons.org/licenses/by/ $4.0 /)$.

\begin{abstract}
In 'Tsuda' turnip, the swollen root peel accumulates anthocyanin pigments in a lightdependent manner, but the mechanism is unclear. Here, mutant $g 120 w$ which accumulated extremely low levels of anthocyanin after light exposure was identified. Segregation analysis showed that the anthocyanin-deficient phenotype was controlled by a single recessive gene. By using bulkedsegregant analysis sequencing and CAPS marker-based genetic mapping analyses, a 21.6-kb region on chromosome A07 was mapped, in which a calcium-binding EF hand family protein named BrLETM2 was identified as the causal gene. RNA sequencing analysis showed that differentially expressed genes (DEGs) between wild type and $g 120 w$ in light-exposed swollen root peels were enriched in anthocyanin biosynthetic process and reactive oxygen species (ROS) biosynthetic process GO term. Furthermore, nitroblue tetrazolium (NBT) staining showed that the ROS level decreased in $g 120 w$ mutant. Anthocyanins induced by UV-A were abolished by the pre-treatment of seedlings with DPI (an inhibitor of nicotinamide adenine nucleoside phosphorylase (NADPH) oxidase) and decreased in g120w mutant. These results indicate that BrLETM2 modulates ROS signaling to promote anthocyanin accumulation in turnip under UV-A and provides new insight into the mechanism of how ROS and light regulate anthocyanin production.
\end{abstract}

Keywords: anthocyanin; bulk segregant analysis; BrLETM2; ROS

\section{Introduction}

Anthocyanins are polyphenolic secondary metabolites that are ubiquitous in the plant kingdom. They play a role not only in reproduction, by attracting pollinators and seed dispersers, but also in protection against various abiotic and biotic stresses [1]. For consumers, anthocyanin-enriched fruits and vegetables are considered to be healthy and delectable, which are important target traits in horticulture plants breeding programs.

Anthocyanins are produced from a well-characterized pathway that contains key structural genes: CHALCONE SYNTHASE (CHS), CHALCONE ISOMERASE (CHI), FLAVANONE 3-HYDROXYLASE (F3H), DIHYDROFLAVONOL-4-REDUCTASE (DFR), ANTHOCYANIDIN SYNTHASE (ANS), and UDP-GLUCOSE: FLAVONOID 3-O-GLUCOSYLTRANSFERASE (UFGT). The regulation of anthocyanin biosynthesis pathway is mediated by the regulatory MBW complex, in which R2R3-MYB, basic helix-loop-helix (bHLH), and WD40-repeat (WDR) transcription factors (TFs) are involved [2]. In Arabidopsis, the key components of the complex-R2R3-MYB protein PRODUCTION OF ANTHOCYANIN PIGMENT1 
(PAP1) and bHLH protein TRANSPARENT TESTA 8 (TT8)—are able to directly bind the promoter region of anthocyanin biosynthetic genes such as DFR and ANS and activate their expression [3-5]. Both R2R3-MYB and bHLH TFs in many species are regulated by various environmental stimuli to induce anthocyanin biosynthesis in a temporal and spatial manner [6-8].

Light is one of the most important environmental inducers of anthocyanin accumulation $[9,10]$. The regulation mechanism of the MBW complex by light is complicated. Light suppresses a negative regulator CONSTITUTIVE PHOTOMORPHOGENIC1 (COP1) to stabilize light-responsive factors, including ELONGATED HYPOCOTYL 5 (HY5), B-box proteins (BBXs), PAP1 and PAP2, for upregulating anthocyanin biosynthesis [11,12]. On the other hand, HY5 and BBXs are thought to be involved in the light-induced anthocyanin pathway by activating the R2R3-MYB regulators and biosynthetic genes of anthocyanin pathways $[13,14]$. Moreover, the reactive oxygen species (ROS) is reported to participate in light-induced anthocyanin accumulation [15-17]. Studies of ROS inhibitor diphenyleneiodonium chloride (DPI) and ROS inducer paraquat have revealed that the repression of NADP oxidase activity leads to decreased anthocyanin levels, while induction of ROS generation triggers anthocyanin accumulation by activating the regulatory genes PAP1 and TT8 in Arabidopsis [10,18]. Anthocyanin-deficient mutants had relatively higher ROS levels than wild type after paraquat treatment, indicating that anthocyanins function as antioxidants to protect against ROS in plants. Transcriptome analysis of seedlings grown in the dark, light, or treated with $\mathrm{H}_{2} \mathrm{O}_{2}$ showed that a high percentage of $\mathrm{H}_{2} \mathrm{O}_{2}$-responsive genes are also regulated by light, which suggests that light induces $\mathrm{H}_{2} \mathrm{O}_{2}$ signaling in Arabidopsis [19]. During de-etiolation, HY5 induces some ROS-responsive gene expression to scavenge singlet oxygen that generated by photosensitized protochlorophyllide under light [20]. Although light is known to affect ROS production, the mechanism by which light regulates ROS production and anthocyanin accumulation is poorly understood.

Turnip (Brassica rapa subsp. rapa) is one of the most important horticultural vegetables that is widely distributed throughout the world. The swollen root of turnip displays rich morphotypes such as size, shape, and color during domestication. White, green, red, and purple turnip cultivars have been found, which provide enriched nutrition that benefits human health $[21,22]$. The purple cultivar Tsuda from Japan is enriched in anthocyanins in the peels of its swollen root when exposed to light. Previous studies showed that UVA irradiation or co-irradiation with blue and UV-B (blue + UV-B) are the most efficient wavelengths for promoting anthocyanins accumulation in the peels of swollen roots of 'Tsuda' $[23,24]$. Transcriptome analysis identified expression patterns of light-responsive genes and anthocyanin biosynthetic genes in response to UV-A and Blue + UV-B, indicating that an overlapped signaling transduction regulatory network may exist to regulate anthocyanin accumulation. Furthermore, ROS-scavenging pathway genes were upregulated after UV-A irradiation [24]. The mechanism of how UV-A regulates anthocyanins of swollen root peel, however, also remains unclear.

Toward understanding how UV-A regulates anthocyanin accumulation in turnip, we previously constructed a T-DNA library by an improved Agrobacterium-mediated vacuum infiltration method [25]. We screened mutants with abnormal anthocyanin traits of swollen root peel in $T_{2}$ generation and identified desirable mutants that displayed a heritable phenotype in $\mathrm{T}_{3}$ generation. Among them, we found an anthocyanin-deficient mutant, g120w. In the present study, we identified BrLETM2 as the candidate gene for the mutation in $g 120 w$ using a map-based cloning strategy, combined with a bulkedsegregant analysis (BSA) and a modified BSA-seq method. The transcriptome analysis and histochemical staining results indicated that BrLETM2 is involved in light-induced anthocyanin accumulation by promoting ROS levels in turnip. 


\section{Results}

\subsection{Phenotypic Characterization and Genetic Analysis of g120w Mutant}

In Tsuda turnip, light-exposed swollen root peel accumulates large amounts of anthocyanins. The g120w mutant was identified from a $\mathrm{T}_{2}$ population of our previous T-DNA insertion library [25]. However, we failed to amplify fragments of reporter genes of the insertion vector in $g 120 w$, which may be due to a loss of T-DNA insertion during reproductive generation, but it still displayed a heritable anthocyanin-deficiency phenotype.

When exposed to light, the swollen root peel of $g 120 w$ showed reduced purple pigmentation compared to the wild type (Figure 1A) and measurements of anthocyanin showed that $g 120 w$ had accumulated less anthocyanins in swollen root peel (Figure 1B). Consistent with the anthocyanin-deficiency phenotype, the expression level of anthocyaninbiosynthetic genes such as $B r C H S 1, B r D F R$, and BrANS1, and regulatory factors BrPAP1.3 and BrTT8 were robustly decreased in $8120 w$ (Figure 1C-G).

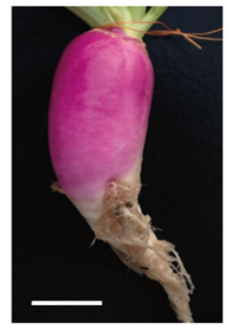

WT

C
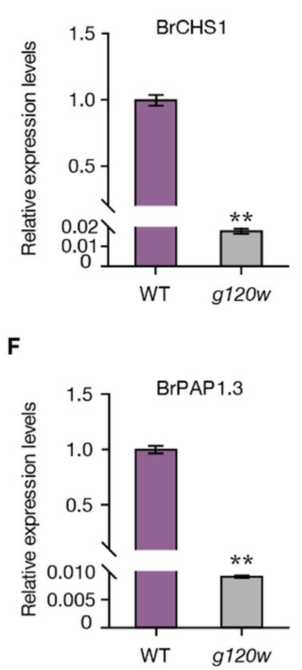

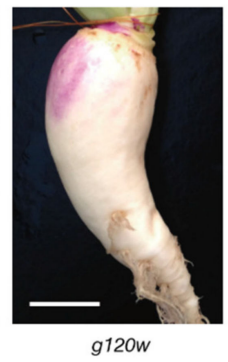

D

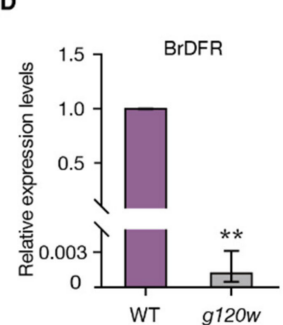

G

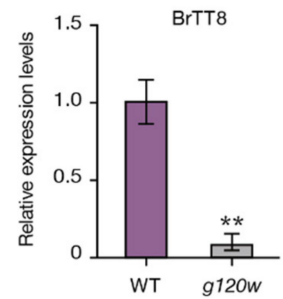

B

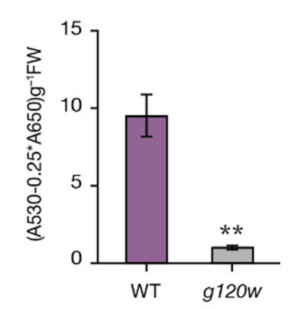

E

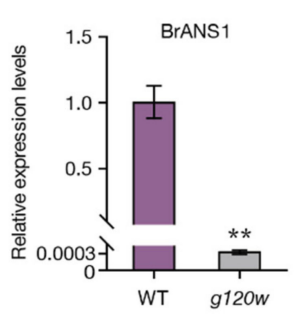

Figure 1. Characterization of $g 120 w$ mutant. (A) Phenotypic variation in the swollen root of the wild type and $g 120 w$. Bars $=5 \mathrm{~cm}$. (B) Anthocyanin content in wild type and $g 120 w$ swollen root peel. (C-G) Quantitative real-time PCR (qRT-PCR) analysis of BrCHS1, BrDFR, BrANS1, BrPAP1.3, and $B r T T 8$ expression levels in swollen root peels in (A). Mean $( \pm \mathrm{SD})$ expression levels were normalized by BrACTIN7, and results for wild type were set at 1 . Asterisks indicate a significant difference between WT and $g 120 w\left({ }^{* *} p<0.01\right.$, Student's $t$-test, $n=3$ ).

To characterize the inheritance of $g 120 w$, we used $g 120 w$ as the maternal plant in a cross with the wild type and generated the $\mathrm{F}_{1}$ population, which was self-pollinated to generate the $F_{2}$ population. Phenotypic segregation analysis showed that the $F_{1}$ progeny had a purple peel. For the $270 \mathrm{~F}_{2}$ individuals, 204 had purple peels (PP-type) and 66 had white peels (WP-type), which was consistent with the predicted 3:1 segregation $\left(\chi^{2}=0.833\right)$. The genetic analysis of $g 120 w$ showed that the anthocyanin deficiency trait was caused by a single recessive gene. 


\subsection{Identification of Candidate Gene by BSA-seq}

For identifying the gene responsible for the low anthocyanin pigmentation in $g 120 w$, four DNA libraries, two parental lines (WT, g120w) and two bulked $\mathrm{F}_{2}$ populations (PP-type and WP-type) generated by the Illumina HiSeqTM2500 platform were used for BSA-seq analysis. A $\Delta$ (SNP-index) comparison between DNA pools of the PP-type and WP-type progeny revealed a significantly different pattern on chromosome A07 in the region from $19.00 \mathrm{Mb}$ to $21.86 \mathrm{Mb}$ associated with the $g 120 \mathrm{w}$ locus (Figure $2 \mathrm{~A}$ ). In the candidate region, 18 SNPs that caused nonsynonymous variations in the coding region of 15 genes were identified (Table S1).

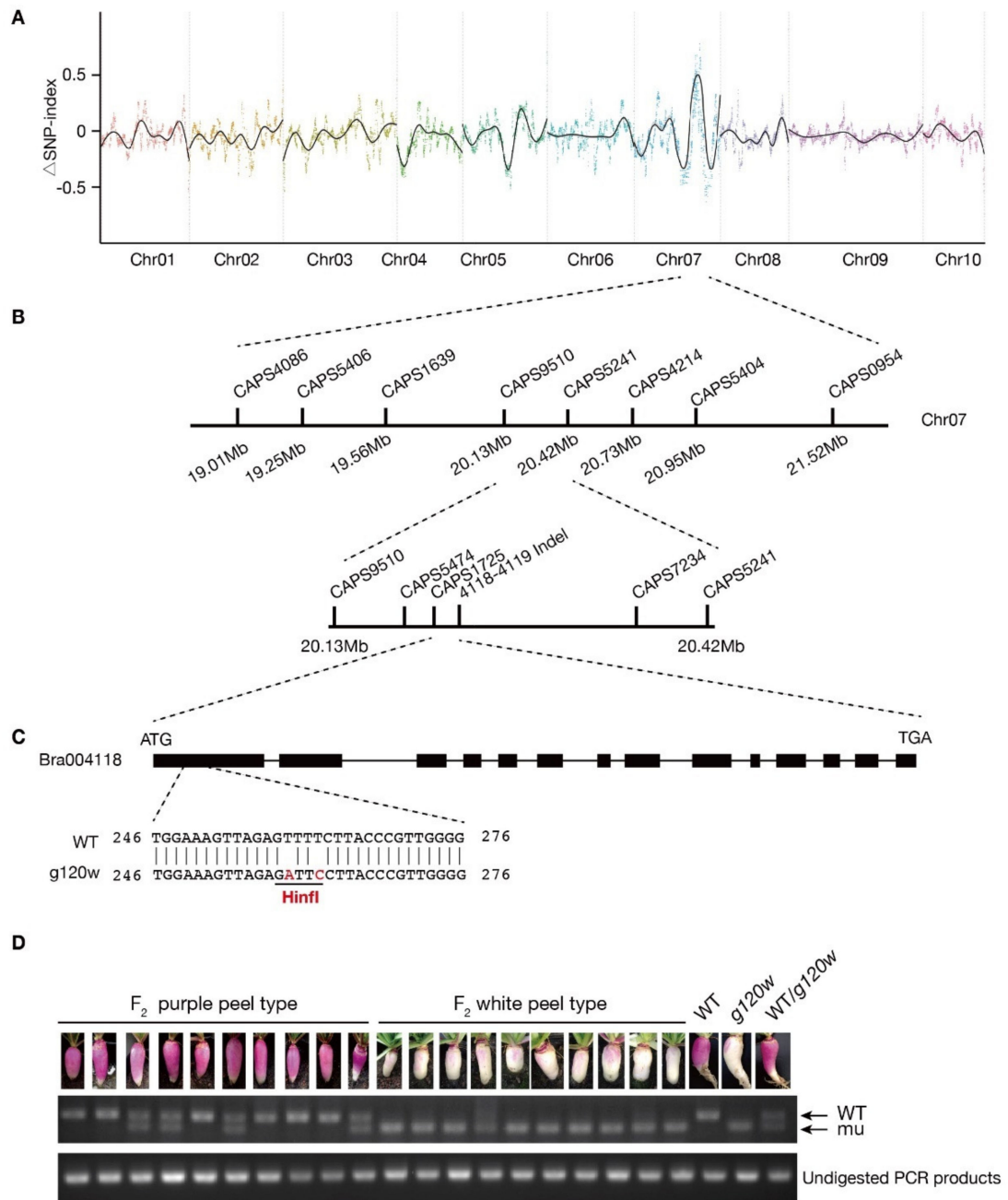

Figure 2. Map-based cloning of $g 120 w$. (A) The calculation of $\Delta$ SNP-index values on chromosomes to identify the candidate regions that associated with the anthocyanin deficiency trait between the two segregate bulks of BSA-Seq (PP-type and WP-type). The curved black lines are average $\Delta$ (SNP-index) values. $X$-axis, physical position; $Y$-axis, SNP index. (B) Genetic mapping of the candidate gene using CAPS markers. (C) Diagram of structure of BrLETM2 gene and amino acid variation of BrLETM2 protein between wild type and $g 120 w$. Black box indicates exons. (D) Genotypic analysis of CAPS1725 in first exon of BrLETM2. Two nucleotide variation led to digestion of fragments in $g 120 w$ by restriction enzyme Hinf1. All the detected $\mathrm{F}_{2}$ progeny with the wild type or mutant phenotype co-segregated with CAPS1725.

To narrow the candidate region in the $g 120 w$ locus, 10 CAPS markers and one indel marker within the candidate region were developed for fine mapping (Figure 2B). Five recombinant plants of WP-type $\mathrm{F}_{2}$ progenies were used to the narrow the candidate area in $g 120 w$ to a $21.8-\mathrm{kb}$ region, between the markers CAPS5474 and 4119INDEL, in which only contains one candidate gene Bra004118. From a functional annotation analysis, Bra004118, 
which encodes a calcium-binding EF hand family protein, was designated as BrLETM2 since it had a high similarity to AtLETM2 in Arabidopsis. To identify variations in BrLETM2 gene, the full length of BrLETM2 was cloned from the swollen root peel of wild type and g120w. A sequence alignment showed that the BrLETM2 from g120w contains a sevenamino-acid variation and one amino acid insertion in the first exon (Figure S1). Then, a CAPS1725 marker was designed to confirm an association between the genotypic variation in BrLETM2 with anthocyanin deficiency. The BrLETM2 gene in g120w had two nucleotide variations compared with wild type, which could be specifically digested by restriction enzyme HinfI in the PCR-amplified fragment of CAPS1725 (Figure 2C). The genotypic assay showed that CAPS1725 co-segregated with the anthocyanin deficiency phenotype in $\mathrm{F}_{2}$ population (Figure 2D). Therefore, BrLETM2 is presumed as the candidate gene in $g 120 \mathrm{w}$.

\subsection{Spatiotemporal Transcription and Phylogenetic Analyses of BrLETM2}

The expression of BrLETM2 was then quantified in various organs in wild type and g120w using qRT-PCR. In wild type, the highest expression of BrLETM2 was in the flowers, followed by the root peels, cotyledon, and silique. The lowest BrLETM2 expression levels were in the leaves and hypocotyls (Figure 3A). Except for flowers, the expression of BrLETM2 in other organs was lower in g120w than those in the wild type. In a phylogenetic tree to elucidate the evolutionary relationships of BrLETM2 with other LETMs orthologs of various species, BrLETM2 clustered with various dicots and was closely related to AtLETM2 (Figure 3B).

A

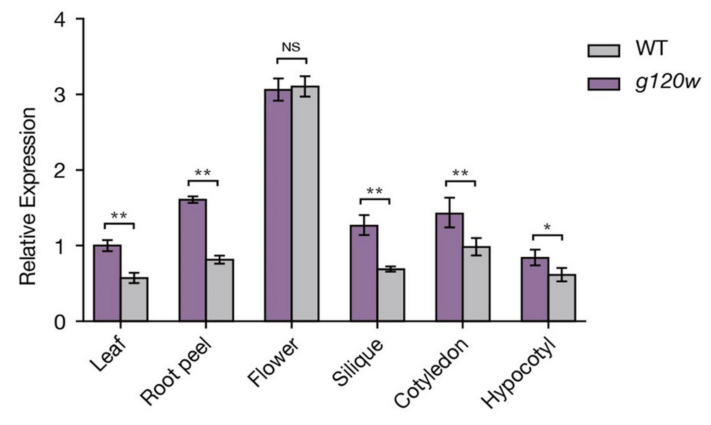

B

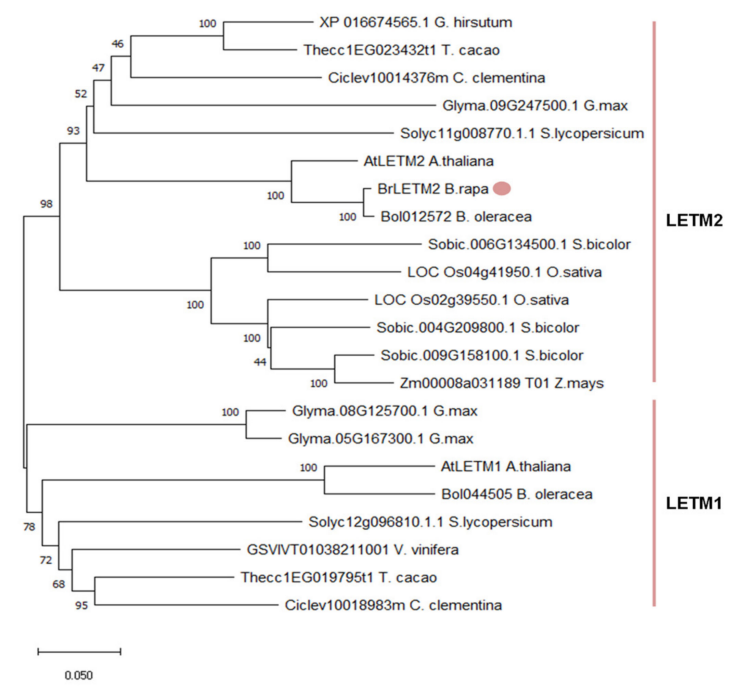

Figure 3. Expression analysis of BrLETM2 in wild type and phylogenetic tree of LETM2 proteins among different plant species. (A) qRT-PCR analysis of BrLETM2 expression in wild type and $g 120 w$ in different organs. Values are means \pm SD $(n=3)$; asterisks indicate a significant difference between wild type and $g 120 w$ among organs by Student's $t$-test $\left({ }^{*} p<0.05\right.$, $\left.{ }^{* *} p<0.01\right)$. (B) Phylogenetic tree constructed using the neighbor-joining method for LETM protein sequences. The tree is drawn to scale, and branch lengths indicate evolutionary distance. 


\subsection{Ectopic Expression of the BrLETM2 Promotes Anthocyanin Accumulation in Arabidopsis}

To verify whether BrLETM2 participates in anthocyanin biosynthesis, the full length of BrLETM2 from WT and g120w were cloned into pCMP1-35S vector and separately inserted into Col-0 Arabidopsis using an Agrobacterium-mediated flower dipping method. $B r L E T M 2^{W T}$-overexpressing $(O E)$ and BrLETM2 ${ }^{m u}-O E$ seedlings accumulated extremely low levels of anthocyanin as Col-0 under control condition, while high sucrose-grown seedlings displayed a dwarf and enriched anthocyanin phenotype (Figure 4A). The BrLETM2 ${ }^{W T}$. $O E$ seedlings accumulated more anthocyanins than the wild type and BrLETM2 ${ }^{m u_{-} O E}$ seedlings when treated with high sucrose (Figure 4B,C). The mRNA of BrLETM2 was significantly induced at a comparable level in BrLETM2 ${ }^{\text {WT }}-O E$ and BrLETM2 ${ }^{m u}-O E$ seedlings (Figure 4D). The anthocyanin biosynthetic genes AtCHS, AtDFR, AtLDOX, and AtPAP1 were more highly expressed in BrLETM2 ${ }^{W T}$-OE seedlings than those of in $B r L E T M 2^{m u_{-} O E}$ and wild type, but not AtTT8 (Figure 4E-I).

A

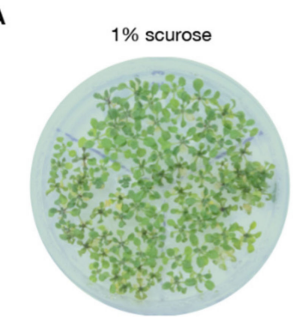

B

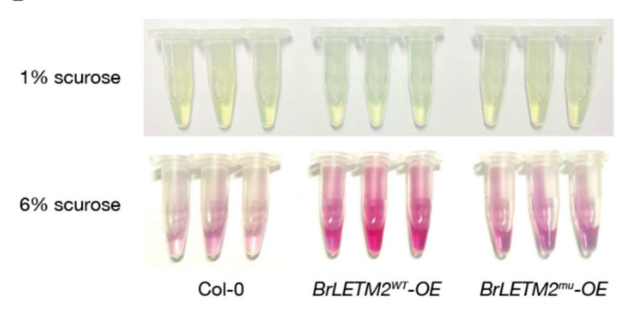

D

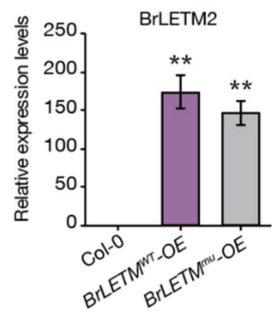

G

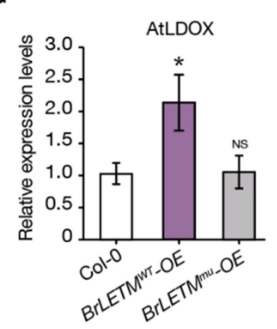

E

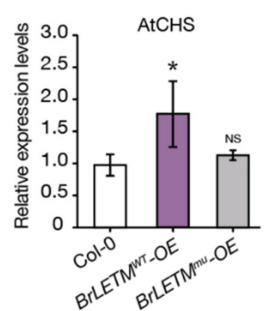

H

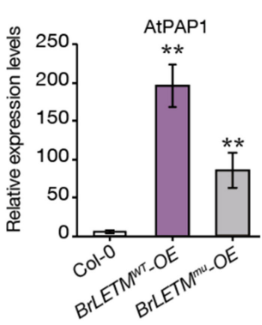

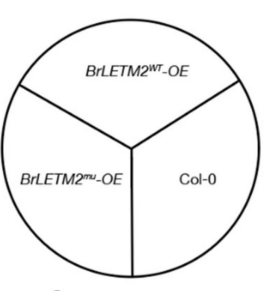

C

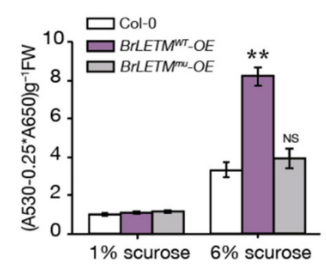

F
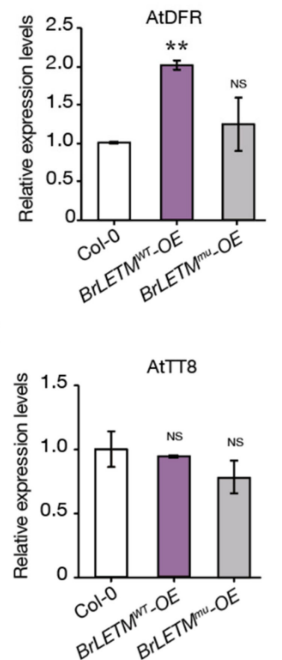

Figure 4. BrLETM2 promotes anthocyanin biosynthesis in response to high sucrose in 14-day-old seedlings of Arabidopsis. (A) Seedlings of Col-0, BrLETM2 ${ }^{W T}-O E$ and $B r L E T M 2^{m u}-O E$ were grown on plates with normal sucrose (1\%) and high sucrose (6\%). (B,C) Anthocyanin levels of seedlings in (A). (D-I) Expression levels of BrLETM2, AtCHS, AtDFR, AtLDOX, AtPAP1, and AtTT8 in seedlings in (A) were quantified by qRT-PCR. Expression level of Col-0 was set at 1 and AtACTIN8 was used for normalization. Values are means $\pm \mathrm{SD}(n=3)$; asterisks indicate a significant difference between Col-0 and overexpression lines $\left({ }^{*} p<0.05,{ }^{* *} p<0.01\right.$, Student's $t$-test). 


\subsection{RNA-seq Analysis Revealed Enrichment of the Anthocyanin and ROS Signaling Pathway in $g 120 w$}

To understand how BrLETM2 regulates anthocyanin biosynthesis, RNAs from lightexposed swollen root peel of wild type and $g 120 w$ were sequenced and analyzed. Deep sequencing yielded 59 to 75 million reads from the wild type and g120w samples. After quality control, $84 \%$ of the reads were mapped to the reference genome. A correlation coefficient analysis and PCA showed that the biological replicates of $g 120 w$ and wild type samples can be well-clustered (Figure S2).

For the anthocyanin-deficiency phenotype of $g 120 w$, candidate genes involved in anthocyanin biosynthetic pathway were screened and analyzed. Multiple copies of the anthocyanin biosynthetic genes (ABGs) were present in turnip and differentially expressed in the swollen root peel of wild type and $g 120 \mathrm{w}$. The transcript levels of anthocyanin biosynthetic genes BrPAL1.1, BrPAL2.1, BrC4H5, Br4CL3, BrCHS1, BrCHS3, BrCHI1, BrF3H1, $B r D F R, B r A N S 1$, and BrUF5GT were much lower in $g 120 w$ than in the wild type, and the expression of the key regulatory factors BrPAP1.3 and BrTT8 was also reduced (Figure 5A).

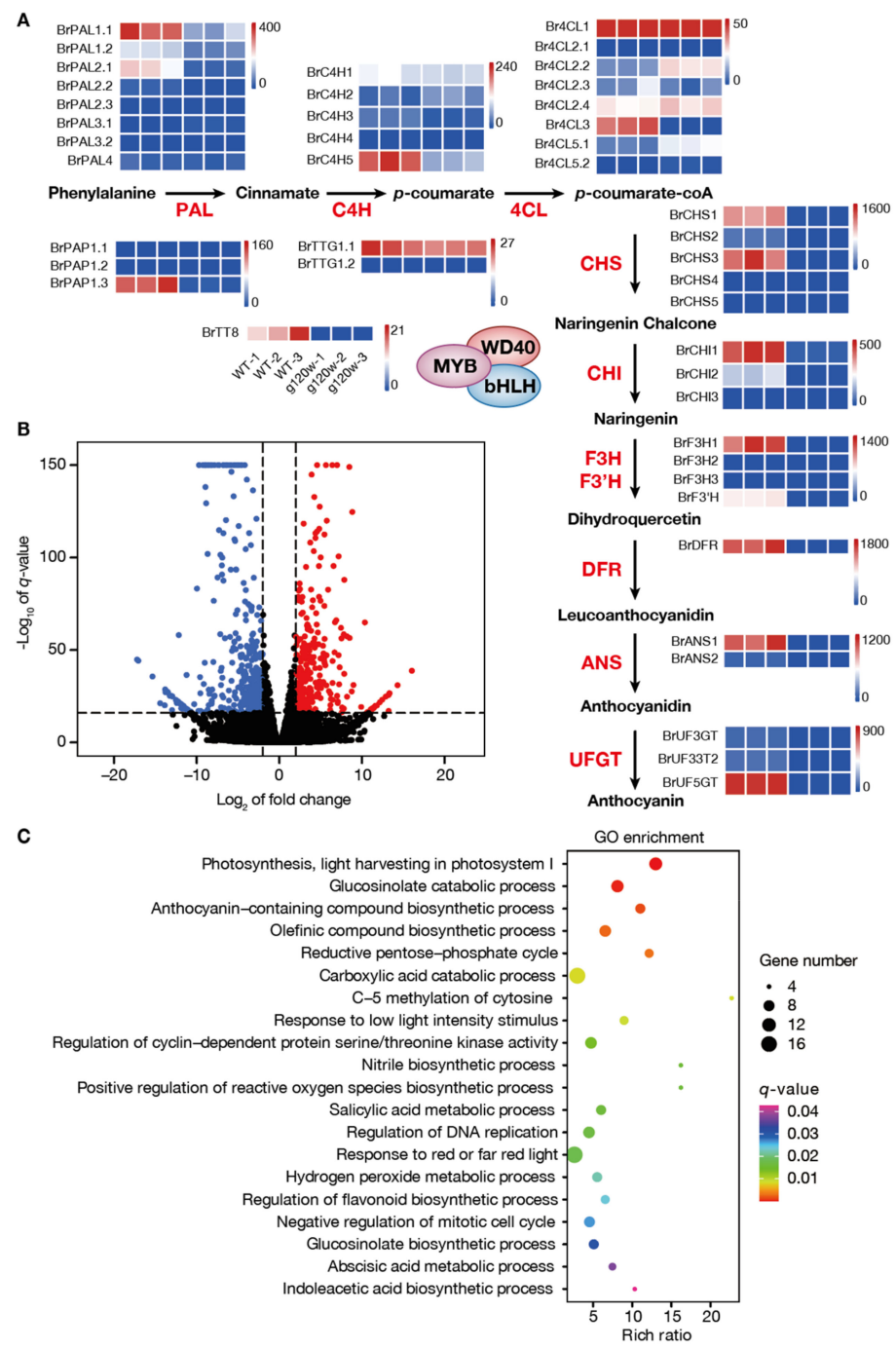

Figure 5. Function annotation of differentially expressed genes (DEGs) between wild type and g120w. (A) Schematic of anthocyanin biosynthetic pathway and mRNA levels of anthocyanin-related genes in wild type and g120w based on RNA-seq. (B) The volcano plot of DEGs from wild type and g120w. The blue spots represent downregulated DEGs, and the red spots represent upregulated DEGs. (C) GO enrichment analysis of DEGs between wild type and $g 120 w$ in biological process. 
A total of 3268 genes were differentially expressed (DEGs) in the root peels of wild type and $g 120 w$, of which 1728 were upregulated and 1540 were downregulated in wild type (Figure 5B). To identify the BrLETM2-induced genes, we evaluated upregulated DEGs in GO enrichment analyses. Besides genes involved in anthocyanin biosynthesis, the upregulated DEGs were also enriched in response to light stimulation, positive regulation of the reactive oxygen species (ROS) biosynthesis process and hydrogen peroxide metabolism process (Figure 5C). Consistent with their differences in anthocyanin accumulation, the ROS level of swollen root peel in g120w was lower than in the wild type (Figure S3), indicating that BrLETM2 may regulate anthocyanin accumulation partially through the ROS signaling pathway.

\subsection{BrLETM2 Promotes ROS Levels of Turnip Seedlings under UV-A}

As an inner mitochondrial membrane protein, LETM is responsible for mitochondrial $\mathrm{Ca}^{2+}$ levels, which affects ROS levels in cells $[26,27]$. ROS can promote anthocyanin accumulation in Arabidopsis [18]. Our previous studies showed that ROS signaling also participates in UV-A induced the anthocyanin accumulation process in swollen root peel [24]. Meanwhile, the expression of BrLETM2 was also increased in response to UV-A irradiation (Figure S4). To validate the role of ROS in regulating the anthocyanin biosynthesis under UV-A irradiation, wild type seedlings were pretreated with diphenylene-iodonium chloride (DPI), an inhibitor of NADPH oxidase, for $2 \mathrm{~h}$ before exposure to UV-A. Anthocyanin levels of DPI-treated seedlings were robustly reduced after $24 \mathrm{~h} \mathrm{UV}$-A treatment compared with $\mathrm{H}_{2} \mathrm{O}$-treated seedlings, indicating that ROS participates in the UV-A induced anthocyanin accumulation (Figure 6A,B). The anthocyanin level in the hypocotyl of $g 120 w$ was significantly lower than in that of the wild type (Figure 6A,B). To elucidate whether BrLETM2 regulates ROS levels, we used nitroblue tetrazolium chloride (NBT) as a histochemical stain. Seedlings of the wild type and $g 120 w$ were exposed to UV-A for $24 \mathrm{~h}$, then the levels of superoxide $\left(\mathrm{O}_{2}{ }^{-}\right)$were detected by NBT (Figure 6C). The results showed that the $\mathrm{O}_{2}{ }^{-}$ levels in the hypocotyls of $g 120 w$ were significantly lower than in those of the wild type. The mRNA level of the ROS-responsive genes BrCAT2 (Catalase-2) and BrPOD (Peroxidase 31) were induced by UV-A and inhibited in DPI-pretreated seedlings (Figure 6D,E). Meanwhile, BrLETM2 exhibited a similar expression pattern as ROS-responsive genes, indicating that BrLETM2 is involved in ROS production under UV-A irradiation (Figure 6F). Furthermore, $B r C A T 2$ was significantly decreased in g120w compared with that of the wild type, which is consistent with decreased $\mathrm{O}_{2}{ }^{-}$levels in $g 120 w$ seedlings. In contrast, no difference of BrPOD and BrLETM2 expression level between wild type and $g 120 w$ was observed after UV-A irradiation. These results together suggest that BrLETM2 promotes ROS production to induce anthocyanin accumulation after turnip is exposed to UV-A. 

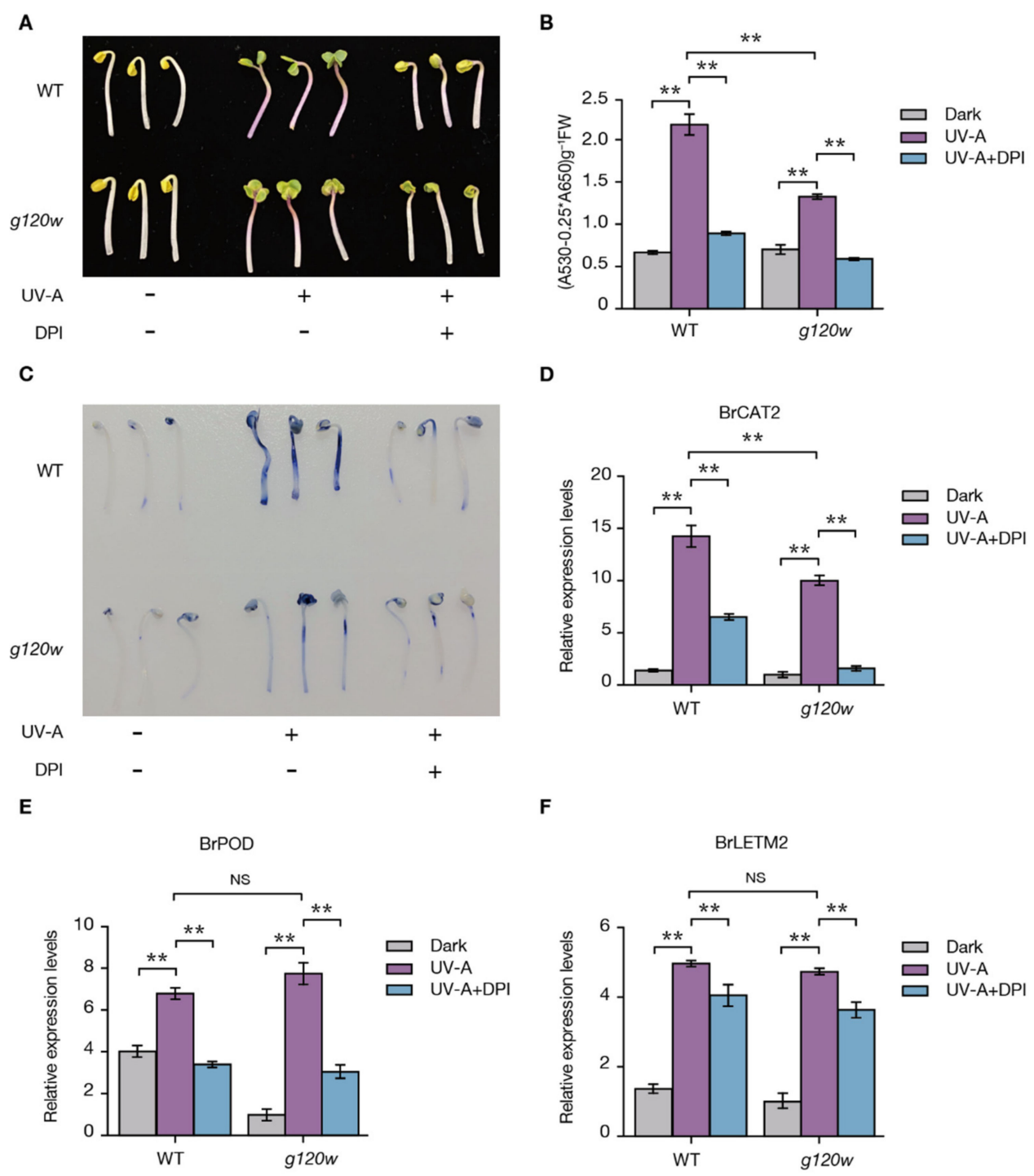

Figure 6. BrLETM2 is a positive regulator of ROS production and anthocyanin accumulation under UV-A irradiation. (A) Effects of UV-A and DPI on the morphology of turnip sprouts. (B) Anthocyanin levels of hypocotyls in (A). (C) NBT staining of turnip sprouts in (A). (D-F) qRT-PCR analysis of BrCAT2, BrPOD, and BrLETM2 expression levels in hypocotyls in (A). Mean ( \pm SD) expression levels were normalized by BrACTIN7, and results for $g 120 w$ in darkness were set at 1 . Asterisks indicate significant difference between different treatments in WT and $g 120 w{ }^{* *} p<0.01$, Student's $t$-test, $n=3$ ).

\section{Discussion}

Ultraviolet light is a key environmental signal perceived by plants that affects the flavonoid pathway and influences the levels of anthocyanins, flavonols, and proanthocyanidins $[16,28,29]$. As high light can induce ROS production, and ROS are also an important signal that can induce anthocyanin accumulation, studies have been conducted to identify the regulators underlying anthocyanin pigmentation mediated by ROS under solar UV radiation [30,31]. In this study, starting from the genetic analysis of the turnip mutant with anthocyanin-deficiency phenotype, we identified an LETM protein that controls the anthocyanin pigmentation of turnip swollen root peel and elucidated the mechanism of its action after UV-A exposure. We demonstrated that BrLETM2 is a positive regulator of anthocyanin pigmentation, which can be induced by UV-A and promote the ROS production. Hence, we speculate that the BrLETM2 protein modulates anthocyanin accumulation by promoting ROS production after UV-A exposure. 
The LETM gene encodes a mitochondrial inner membrane protein, which is highly conserved across a broad range of eukaryotic organisms [32]. To date, there are only two reports about the function of LETM in plants. One showed that AtLETM1 and AtLETM2 are important proteins for the accumulation of ATP synthase in Arabidopsis [33]. The other showed that GhLETM1 plays a critical role in regulating stamen development and male fertility in cotton (Gossypium hirsutum L.) [34]. The primary functional characteristics of LETM identified in animals shows that LETM proteins act as an $\mathrm{Ca}^{2+} / 2 \mathrm{H}^{+}$antiporter [32], and mitochondrial $\mathrm{Ca}^{2+}$ influx promotes ROS generation [26]. Meanwhile, because mitochondria are considered as the main source of ROS in the cell, ectopic expression of LETM1 in HeLa cells changed the membrane potential, consequently leading to increased ROS production [27]. By histochemical staining of the hypocotyl with ROS indicator, we also found that the mutation in BrLETM2 led to decreased ROS production in $8120 w$ (Figure 6C), which implies that LETM may work in a similar way to that in animals to enhance the ROS generation in plants.

High-intensity UV-B causes damage to DNA, proteins, and membranes in plants, but low-intensity UV-B, normal UV-A, and high-intensity visible light (usually referred to as HL) can promote ROS production and anthocyanin accumulation $[15,16]$. Previously, we found that genes induced by UV-A were significantly enriched for GO categories related to the ROS signaling pathway in turnip peels [24]. In the present work, after pretreatment with DPI, the ROS level decreased under UV-A irradiation (Figure 6C), indicating that the ROS signals may integrate with the UV-A light signal pathway to modulate anthocyanin accumulation. However, we also found that the NBT staining in the hypocotyl of seedling of g120w was not completely lost (Figure 6C), and anthocyanin pigmentation was also not completely lost in the swollen root peels (Figure 1A,B). Considering that the photoreceptors and light signal transduction pathways are crucial for light-induced anthocyanin accumulation [9,35], it is reasonable to speculate that the LETM-mediated ROS signaling pathway only acts as a secondary messenger in this process.

The anthocyanin structural genes and regulatory genes in $B$. rapa have been identified by comparative analysis of genomic sequences of B.rapa with $A$. thaliana [36]. Most of the ABGs in $B$. rapa exist in multiple copies, indicating that these ABGs would likely function redundantly and determine the anthocyanin accumulation of B.rapa species in a spatial and temporal manner. However, the key ABGs in regulating anthocyanin biosynthesis in the peels of the swollen root of turnip have not been characterized. Based on our RNA-seq data, we identified a series of candidate genes that may contribute to anthocyanin accumulation in swollen root peel, which showed high expression levels in WT and a robust reduction in $g 120 w$ (Figure 5A). Although other ABGs were not expressed in root peel, they may have functions in response to specific stimuli or in other organs that need to be further investigated.

MBW transcriptional regulators are generally conserved in regulating the expression of anthocyanin biosynthetic genes in eudicots $[37,38]$. We found that the mRNA levels of BrLETM2 and BrPAP1.3 genes can be induced after UV-A irradiation (Figure 6F, Figure S4), but the mRNA level of BrPAP1.3 dramatically decreased in the g120w mutant (Figure 1F). The ectopic expression of BrLETM2 induced AtPAP1 but not AtTT8 in Arabidopsis (Figure $4 \mathrm{H}, \mathrm{I})$. Xu et al. reported that ROS induce the production of anthocyanin by upregulating the LBGs due to the upregulation of AtPAP1 and AtTT8 in Arabidopsis [18]. These results indicate that BrLETM2 may work upstream of BrPAP1.3 and active expression of BrPAP1.3 by ROS signaling to promote anthocyanin accumulation. Although the mRNA level of BrLETM2 can be induced by UV-A, the detailed mechanism remains unknown. Whether this process is mediated by the photoreceptors or light signal transduction factors such as COP1 and HY5 needs to be confirmed with further research. 


\section{Materials and Methods}

\subsection{Plant Materials and Growth Conditions}

Tsuda turnip was developed at the Institute of Flower Bioengineering in Northeast Forestry University. The wild type and $g 120 \mathrm{w}$ and $\mathrm{F}_{2}$ population of a cross between the two lines were grown in a greenhouse. Two months after sowing, when the roots had begun to swell, epidermal peels were used to measure anthocyanins and extract DNA/RNA. Turnip seedlings were grown on filter paper. For UV-A light $(360 \mathrm{~nm})$ treatment, etiolated seedlings were kept in darkness for two days, then transferred to UV-A $\left(3 \mathrm{~W} \mathrm{~m}^{-2}\right)$ for $24 \mathrm{~h}$. Hypocotyls of these seedlings were used for further analysis.

For Arabidopsis, BrLETM2 ${ }^{W T}-O E$ and BrLETM2 ${ }^{m u}-O E$ transgenic lines were derived from Col-0 ecotype. Seedlings were grown on Murashige and Skoog medium for 14 days. Mature plants were grown in a plant growth room with a 16-h light $\left(22\right.$ to $\left.24^{\circ} \mathrm{C}\right) / 8$-h dark $\left(17\right.$ to $\left.19^{\circ} \mathrm{C}\right)$ cycle.

\subsection{Anthocyanin Measurement}

Samples were weighed and ground to a powder in liquid nitrogen, then suspended in $1 \mathrm{~mL}$ of methanol containing $1 \%(v / v) \mathrm{HCl}$ and incubated at $4{ }^{\circ} \mathrm{C}$ for $24 \mathrm{~h}$. The solution was centrifuged at $14,000 \times g$ for $5 \mathrm{~min}$. Then, $0.2 \mathrm{~mL}$ of each supernatant was transferred to a well in a 96-well plate, and the absorbance of samples at $530 \mathrm{~nm}$ (A530) and $650 \mathrm{~nm}$ (A650) was recorded using a microplate reader (TECAN). Anthocyanin concentrations were determined using the following equation: (A530 - 0.25* A650)/Fresh weight (g).

\subsection{BSA-seq Analysis and Phenotype Association Assay}

Total DNA was extracted from leaves by a modified CTAB protocol. In brief, samples were ground into power by liquid nitrogen and put into $2 \%$ CTAB solution ( $2 \%$ CTAB, $100 \mathrm{mM}$ Tris-HCL, $20 \mathrm{mM}$ EDTA, $1.4 \mathrm{mM} \mathrm{NaCl}$ ). Then, samples were purified by P-buffer (phenol: chloroform: isoamylol $=25: 24: 1$ ) twice and precipitated by isopropanol. After centrifuging, the DNA pellet was washed by $70 \%$ ethanol and eluted by sterile water.

For two parental DNA pools, leaves from 10 wild type and $10 \mathrm{~g} 120 \mathrm{w}$ mutant plants were mixed separately and used for WT and g120w libraries. For two $\mathrm{F}_{2}$ DNA pools, equal amounts of DNA from 30 PP-type and $30 \mathrm{WP}$-type $\mathrm{F}_{2}$ individuals were used for PPbulk and WP-bulk libraries. BSA-seq data of four libraries were generated by paired-end sequencing with an Illumina HiSeq 2500 (Illumina Inc., San Diego, CA, USA) NGS platform. Trimmomatic software and FASTQC software were used to obtain clean reads and filter high-quality reads. Filtered clean reads from four libraries were separately mapped to the Brassica rapa v1.5 reference genome (http:/ / brassicadb.org/brad/index.php (accessed on 28 December 2019)) using BWA software. All SNP variation information was generated by GATK2.7 software [39]. Informative SNPs were filtered by comparison of PP-bulk and WP-bulk pools with SAM tools for further SNP-index analysis [40]. The SNP-index of each SNP position from PP-bulk and WP-bulk was calculated; a $\triangle$ SNP-index was generated by subtraction of the WP-bulk SNP index from the PP-bulk SNP index. The $\triangle \mathrm{SNP}$-index across chromosomes of the $B$. rapa genome was performed by sliding-window analysis with a $1 \mathrm{Mb}$ window size and $10 \mathrm{~kb}$ step size. The gene functions were annotated with ANNOVAR [41].

To confirm mutant loci, CAPS markers in the candidate region that may be linked with the mutated genes were screened based on SNP data from BSA-seq analysis and confirmed by co-segregation analysis. A total of 270 recessive mutant $\mathrm{F}_{2}$ plants were used for fine mapping. A 200 500-bp target fragment with an SNP was amplified by specific primers (Table S2). For enzyme digestion, $5 \mathrm{U}$ of restriction enzyme was added into a tube that contained $8 \mu \mathrm{L}$ PCR products, $1.5 \mu \mathrm{L} 10 \times$ restriction buffer, and water, and incubated at $37^{\circ} \mathrm{C}$ for $3 \mathrm{~h}$. Bands were separated electrophoretically in $2 \sim 5 \%$ agarose. 


\subsection{RNA Isolation and Gene Expression Analysis}

The total RNA was extracted with TRIZol-Reagent (Invitrogen, Carlsbad, CA, USA). The cDNA of samples was generated using TransScript One-Step gDNA Removal and a cDNA Synthesis SuperMix kit (TransGen Biotech, Beijing, China) according to the manufacturer's protocol. The qRT-PCR was performed with SYBR Green PCR master mix (Roche, Rotkreuz, Switzerland). Relative expression levels were calculated by the $2^{-\Delta \Delta \mathrm{Ct}}$ method. The expression of target genes was normalized using the $B r A C T 7$ as a reference gene for turnip and AtACT8 for Arabidopsis. All gene-specific primers used for qRT-PCR analysis are listed in Supplemental Table S2.

\subsection{RNA-seq of Differential Gene Expression}

Samples for RNA-seq were taken from the swollen root peels of wild type and $g 120 w$ mutant grown in sunlight, with three biological replicates for each line. RNA-seq transcriptome libraries were prepared following TruSeqTM RNA sample preparation kit from Illumina, using $1 \mu \mathrm{g}$ of total RNA. Paired-end libraries were sequenced using Illumina NovaSeq 6000 sequencing by Shanghai BIOZERON Co., Ltd., Shanghai, China.

The raw paired-end reads were trimmed and quality controlled using Trimmomatic (parameters SLIDINGWINDOW: 4:15 MINLEN:75). FastQC software was used for statistics and analysis of raw data and clean data. Then, clean reads were separately aligned to the reference genome (Brassica rapa v1.5) with orientation mode using hisat2 software with default parameters [42].

FeatureCounts software v1.6.3 (http:/ / subread.sourceforge.net (accessed on 10 January 2020)) and the fragments per kilobase of exon per million mapped reads (FRKM) method were used to count reads for each gene and calculate the expression level for each gene, respectively. The R statistical package edgeR (http:/ / www.bioconductor.org/ packages/release/bioc/html/edgeR.html/ (accessed on 10 January 2020)) was used for the differential expression analysis. The threshold fold-change $\geq 4$ and FDR $<0.05$ were used to screen for significant DEGs. GO functional enrichment was carried out using Gene Ontology online (http:/ / geneontology.org (accessed on 13 January 2020)) to annotate the functions of the DEGs. DEGs were considered to be significantly enriched in GO terms and metabolic pathways when their Bonferroni-corrected $p$-value was less than 0.05.

\subsection{NBT Staining and ROS Measurement}

$\mathrm{O}_{2}{ }^{-}$levels were detected as previously described with minor modifications [43]. The seedlings were placed in the NBT staining solution containing $1 \mathrm{mg} \mathrm{mL}^{-1} \mathrm{NBT}$ in $50 \mathrm{mM}$ sodium phosphate buffer ( $\mathrm{pH}$ 7.5). The seedlings were then incubated for $6 \mathrm{~h}$ in the dark with gentle shaking. The staining solution was replaced with absolute ethanol in a boiling water bath for $30 \mathrm{~min}$ to remove chlorophyll. The seedlings were transferred to filter paper with $60 \% v / v$ glycerol until images were taken.

Briefly, swollen root peels were ground using a mortar and pestle in buffer $\left(9 \mathrm{~mL} \mathrm{mg}^{-1}\right.$ fresh weight) containing $50 \mathrm{mM}$ Tris- $\mathrm{HCl}$ (pH 7.4). The homogenate was centrifuged at $12,000 \mathrm{rpm}$ for $20 \mathrm{~min}$. The supernatant was transferred into a new tube for measurement. The procedure of ROS detection used was Plant Reactive Oxygen Species (ROS) ELISA Kit (MBS281870) followed the manual as described.

\subsection{Plasmid Construction and Plant Transformation}

The coding sequence (CDS) of BrLETM2 was isolated from the cDNA of swollen root peels of wild type and $g 120 w$ using $2 \times$ Phanta Max Master Mix (Vazyme), and was then separately cloned into pCMP1-35S vector using ClonExpress II One Step Cloning Kit (Vazyme) and individually inserted into Agrobacterium strain GV3101. Plasmids carrying 35S:BrLETM2 ${ }^{\mathrm{WT}}$ and 35S:BrLETM2 ${ }^{\mathrm{mu}}$ were inserted into Arabidopsis thaliana ecotype Col0 using a general flower dip method [44]. Seedlings were screened for the transgene on Murashige and Skoog (MS) medium supplied with $40 \mu \mathrm{g} / \mathrm{mL}$ hygromycin, and the 
recombinants were confirmed by PCR amplification after they were grown in soil for two weeks.

\subsection{Phylogenetic Analysis}

BrLETM2-related proteins in plant species (Figure 3) were identified using a BLASTP search of the phytozome v12.1 database (https:/ / phytozome.jgi.doe.gov/pz/portal.html (accessed on 23 March 2021)) and default parameters. The retrieved sequences were aligned with ClustalW in MEGAX software. The phylogenetic tree was constructed using the neighbor-joining method with default parameters besides 1000 bootstrap replications.

Supplementary Materials: Supplementary Materials can be found at https:/ /www.mdpi.com/ article/10.3390/ijms22073538/s1, Figure S1: Amino acid sequence alignment of BrLETM2 in wild type and g120w, Figure S2: Biological replication analysis of RNA-seq samples, Figure S3: ROS levels of swollen root peel in wild type and g120w, Figure S4: Anthocyanin contents and expression analysis of BrLETM2 and BrPAP1.3 in swollen root peel of wild type after 6h UV-A irradiation, Table S1: List of SNPs within candidate region for g120w mutant, Table S2: Primers used in this study.

Author Contributions: H.D.S. and J.Y. contributed equally to this work. Conceptualization, Y.W., Y.L. and S.K.; methodology, H.D.S., J.Y. and Y.W.; software, B.C. and Y.W.; validation, H.D.S., J.Y., N.H.M., Y.C. and P.K.; formal analysis, H.D.S. and Y.W.; resources, Y.W. and Y.L.; investigation, H.D.S., J.Y., N.H.M., Y.C. and P.K.; data curation, H.D.S., J.Y. and Y.W.; writing, original draft, J.Y. and Y.W.; writing, review and editing the manuscript, H.D.S., J.Y., Y.W. and Y.L.; supervision of all work, Y.W. and Y.L.; project administration, Y.W. and Y.L. All authors have read and agreed to the published version of the manuscript.

Funding: This research was funded by the National Natural Science Foundation of China, grant number 31471911, 31401907, and 31272200, Heilongjiang Touyan Innovation Team Program (Tree Genetics and Breeding Innovation Team) and Fundamental Research Funds for the Central Universities (No. 2572016AA51).

Institutional Review Board Statement: Not applicable.

Informed Consent Statement: Not applicable.

Data Availability Statement: Data are given in the article or in supplementary material.

Acknowledgments: We thank Beth E. Hazen for improving the English of the manuscript.

Conflicts of Interest: The authors declare no conflict of interest.

\section{References}

1. Mahmood, K.; Xu, Z.; El-Kereamy, A.; Casaretto, J.A.; Rothstein, S.J. The Arabidopsis transcription factor ANAC032 represses anthocyanin biosynthesis in response to high sucrose and oxidative and abiotic stresses. Front. Plant Sci. 2016, 7, 1548. [CrossRef]

2. Liu, Y.; Tikunov, Y.; Schouten, R.E.; Marcelis, L.F.M.; Visser, R.G.F.; Bovy, A. Anthocyanin biosynthesis and degradation mechanisms in solanaceous vegetables: A review. Front. Chem. 2018, 6, 52. [CrossRef]

3. Wang, L.; Tang, W.; Hu, Y.; Zhang, Y.; Sun, J.; Guo, X.; Lu, H.; Yang, Y.; Fang, C.; Niu, X.; et al. A MYB/bHLH complex regulates tissue-specific anthocyanin biosynthesis in the inner pericarp of red-centered kiwifruit actinidia chinensis cv. Hongyang. Plant $J$. 2019, 99, 359-378.

4. Li, C.; Qiu, J.; Huang, S.; Yin, J.; Yang, G. AaMYB3 interacts with AabHLH1 to regulate proanthocyanidin accumulation in anthurium andraeanum (Hort.)-another strategy to modulate pigmentation. Hortic. Res. 2019, 6, 14. [CrossRef] [PubMed]

5. Takos, A.M.; Jaffé, F.W.; Jacob, S.R.; Bogs, J.; Robinson, S.P.; Walker, A.R. Light-induced expression of a MYB gene regulates anthocyanin biosynthesis in red apples. Plant Physiol. 2006, 142, 1216-1232. [CrossRef] [PubMed]

6. Cominelli, E.; Gusmaroli, G.; Allegra, D.; Galbiati, M.; Wade, H.K.; Jenkins, G.I.; Tonelli, C. Expression analysis of anthocyanin regulatory genes in response to different light qualities in Arabidopsis thaliana. J. Plant Physiol. 2008, 165, 886-894. [CrossRef] [PubMed]

7. Albert, N.W.; Davies, K.M.; Lewis, D.H.; Zhang, H.; Montefiori, M.; Brendolise, C.; Boase, M.R.; Ngo, H.; Jameson, P.E.; Schwinn, K.E. A conserved network of transcriptional activators and repressors regulates anthocyanin pigmentation in eudicots. Plant Cell 2014, 26, 962-980. [CrossRef] [PubMed]

8. Kiferle, C.; Fantini, E.; Bassolino, L.; Povero, G.; Spelt, C.; Buti, S.; Giuliano, G.; Quattrocchio, F.; Koes, R.; Perata, P. Tomato R2R3-MYB proteins SIANT1 and SIAN2: Same protein activity, different roles. PLoS ONE 2015, 10, e0136365. [CrossRef] 
9. Zoratti, L.; Karppinen, K.; Escobar, A.L.; Haggman, H.; Jaakola, L. Light-controlled flavonoid biosynthesis in fruits. Front Plant Sci. 2014, 5, 534. [CrossRef]

10. Chen, W.; Zhang, M.; Zhang, G.; Li, P.; Ma, F. Differential regulation of anthocyanin synthesis in apple peel under different sunlight intensities. Int. J. Mol. Sci. 2019, 20, 60. [CrossRef]

11. Maier, A.; Schrader, A.; Kokkelink, L.; Falke, C.; Welter, B.; Iniesto, E.; Rubio, V.; Uhrig, J.F.; Hulskamp, M.; Hoecker, U. Light and the E3 ubiquitin ligase COP1/SPA control the protein stability of the MYB transcription factors PAP1 and PAP2 involved in anthocyanin accumulation in Arabidopsis. Plant J. 2013, 74, 638-651. [CrossRef] [PubMed]

12. Podolec, R.; Ulm, R. Photoreceptor-mediated regulation of the COP1/SPA E3 ubiquitin ligase. Curr. Opin. Plant Biol. 2018, 45, 18-25. [CrossRef]

13. Shin, D.H.; Choi, M.; Kim, K.; Bang, G.; Cho, M.; Choi, S.-B.; Choi, G.; Park, Y.-I. HY5 regulates anthocyanin biosynthesis by inducing the transcriptional activation of the MYB75/PAP1 transcription factor in Arabidopsis. FEBS Lett. 2013, 587, 1543-1547. [CrossRef]

14. Gangappa, S.N.; Crocco, C.D.; Johansson, H.; Datta, S.; Hettiarachchi, C.; Holm, M.; Botto, J.F. The Arabidopsis B-box protein BBX25 interacts with HY5, negatively regulating BBX22 expression to suppress seedling photomorphogenesis. Plant Cell 2013, 25, 1243-1257. [CrossRef]

15. Su, N.; Wu, Q.; Liu, Y.; Cai, J.; Shen, W.; Xia, K.; Cui, J. Hydrogen-rich water reestablishes ROS homeostasis but exerts differential effects on anthocyanin synthesis in two varieties of radish sprouts under UV-A irradiation. J. Agric. Food Chem. 2014, 62, 6454-6462. [CrossRef] [PubMed]

16. Zhang, J.; Chen, C.; Zhang, D.; Li, H.; Li, P.; Ma, F. Reactive oxygen species produced via plasma membrane NADPH oxidase regulate anthocyanin synthesis in apple peel. Planta 2014, 240, 1023-1035. [CrossRef] [PubMed]

17. Wu, Q.; Su, N.; Zhang, X.; Liu, Y.; Cui, J.; Liang, Y. Hydrogen peroxide, nitric oxide and UV RESISTANCE LOCUS8 interact to mediate UV-B-induced anthocyanin biosynthesis in radish sprouts. Sci. Rep. 2016, 6, 29164. [CrossRef]

18. Xu, Z.; Mahmood, K.; Rothstein, S.J. ROS induces anthocyanin production via late biosynthetic genes and anthocyanin deficiency confers the hypersensitivity to ROS-generating stresses in Arabidopsis. Plant Cell Physiol. 2017, 58, 1364-1377. [CrossRef]

19. Cheng, H.; Zhang, Q.; Guo, D. Genes that respond to $\mathrm{H}_{2} \mathrm{O}_{2}$ are also evoked under light in Arabidopsis. Mol. Plant. 2013, 6, 226-228. [CrossRef] [PubMed]

20. Chen, D.; Xu, G.; Tang, W.; Jing, Y.; Ji, Q.; Fei, Z.; Lin, R. Antagonistic basic helix-loop-helix/bZIP transcription factors form transcriptional modules that integrate light and reactive oxygen species signaling in Arabidopsis. Plant Cell 2013, 25, 1657-1673. [CrossRef]

21. Podsędek, A. Natural antioxidants and antioxidant capacity of brassica vegetables: A review. LWT Food Sci. Technol. 2007, 40, 1-11. [CrossRef]

22. Chung, I.-M.; Rekha, K.; Rajakumar, G.; Thiruvengadam, M. Production of glucosinolates, phenolic compounds and associated gene expression profiles of hairy root cultures in turnip (brassica rapa ssp. rapa). 3 Biotech, 2016; 6, 175.

23. Zhou, B.; Li, Y.; Xu, Z.; Yan, H.; Homma, S.; Kawabata, S. Ultraviolet A-specific induction of anthocyanin biosynthesis in the swollen hypocotyls of turnip (brassica rapa). J. Exp. Bot. 2007, 58, 1771-1781. [CrossRef] [PubMed]

24. Wang, J.; Wang, Y.; Chen, B.; Kawabata, S.; Li, Y. Comparative transcriptome analysis revealed distinct gene set expression associated with anthocyanin biosynthesis in response to short-wavelength light in turnip. Acta Physiol. Plant. 2016, 38, 134. [CrossRef]

25. Wang, X.; Wang, Y.; Chen, B.; Kawabata, S.; Fang, Z.; Li, Y. Construction and genetic analysis of anthocyanin-deficient mutants induced by T-DNA insertion in 'Tsuda' turnip (brassica rapa). Plant Cell Tissue Organ Cult. 2017, 131, 431-443. [CrossRef]

26. Hwang, M.S.; Schwall, C.T.; Pazarentzos, E.; Datler, C.; Alder, N.N.; Grimm, S. Mitochondrial Ca ${ }^{2+}$ influx targets cardiolipin to disintegrate respiratory chain complex II for cell death induction. Cell Death Differ. 2014, 21, 1733-1745. [CrossRef]

27. Nakamura, S.; Matsui, A.; Akabane, S.; Tamura, Y.; Hatano, A.; Miyano, Y.; Omote, H.; Kajikawa, M.; Maenaka, K.; Moriyama, Y.; et al. The mitochondrial inner membrane protein LETM1 modulates cristae organization through its LETM domain. Commun. Biol. 2020, 3, 99. [CrossRef] [PubMed]

28. Zhou, D.; Zhang, Q.; Li, P.; Pan, L.; Tu, K. Combined transcriptomics and proteomics analysis provides insight into metabolisms of sugars, organic acids and phenols in UV-C treated peaches during storage. Plant Physiol. Biochem. 2020, 157, 148-159. [CrossRef] [PubMed]

29. Li, W.; Tan, L.; Zou, Y.; Tan, X.; Huang, J.; Chen, W.; Tang, Q. The effects of ultraviolet A/B treatments on anthocyanin accumulation and gene expression in dark-purple tea cultivar 'ziyan' (camellia sinensis). Molecules 2020, 25, 354. [CrossRef]

30. Hideg, É.; Jansen, M.A.K.; Strid, Å. UV-B exposure, ROS, and stress: Inseparable companions or loosely linked associates? Trends Plant Sci. 2013, 18, 107-115. [CrossRef] [PubMed]

31. Zhang, N.; Qi, Y.; Zhang, H.J.; Wang, X.; Li, H.; Shi, Y.; Guo, Y.D. Genistein: A novel anthocyanin synthesis promoter that directly regulates biosynthetic genes in red cabbage in a light-dependent way. Front. Plant Sci. 2016, 7, 1804. [CrossRef]

32. Li, Y.; Tran, Q.; Shrestha, R.; Piao, L.; Park, S.; Park, J.; Park, J. LETM1 is required for mitochondrial homeostasis and cellular viability (review). Mol. Med. Rep. 2019, 19, 3367-3375. [CrossRef]

33. Zhang, B.; Carrie, C.; Ivanova, A.; Narsai, R.; Murcha, M.W.; Duncan, O.; Wang, Y.; Law, S.R.; Albrecht, V.; Pogson, B.; et al. LETM proteins play a role in the accumulation of mitochondrially encoded proteins in Arabidopsis thaliana and AtLETM2 displays parent of origin effects. J. Biol. Chem. 2012, 287, 41757-41773. [CrossRef] 
34. Zhang, L.; Zhang, Y.; Fan, Y.; Guo, H.; Guo, H.; Wu, J.; Wang, H.; Zhao, Y.; Lian, X.; Gou, Z.; et al. Ectopic expressions of the GhLETM1 gene reveal sensitive dose effects on precise stamen development and male fertility in cotton. Genes 2020, 11, 772. [CrossRef] [PubMed]

35. Sheerin, D.J.; Hiltbrunner, A. Molecular mechanisms and ecological function of far-red light signaling. Plant Cell Environ. 2017, 40, 2509-2529. [CrossRef]

36. Guo, N.; Cheng, F.; Wu, J.; Liu, B.; Zheng, S.; Liang, J.; Wang, X. Anthocyanin biosynthetic genes in Brassica rapa. BMC Genom. 2014, 15, 426. [CrossRef] [PubMed]

37. Zhang, Y.; Li, Y.; Li, W.; Hu, Z.; Yu, X.; Tu, Y.; Zhang, M.; Huang, J.; Chen, G. Metabolic and molecular analysis of nonuniform anthocyanin pigmentation in tomato fruit under high light. Hortic. Res. 2019, 6, 56. [CrossRef]

38. Montefiori, M.; Brendolise, C.; Dare, A.P.; Lin-Wang, K.; Davies, K.M.; Hellens, R.P.; Allan, A.C. In the solanaceae, a hierarchy of bHLHs confer distinct target specificity to the anthocyanin regulatory complex. J. Exp. Bot. 2015, 66, 1427-1436. [CrossRef] [PubMed]

39. McKenna, A.; Hanna, M.; Banks, E.; Sivachenko, A.; Cibulskis, K.; Kernytsky, A.; Garimella, K.; Altshuler, D.; Gabriel, S.; Daly, M.; et al. The genome analysis toolkit: A MapReduce framework for analyzing next-generation DNA sequencing data. Genome Res. 2010, 20, 1297-1303. [CrossRef] [PubMed]

40. Li, H.B.; Handsaker, A.; Wysoker, T.; Fennell, J.; Ruan, N.; Homer, G.; Marth, G.; Abecasis, R.; Durbin, S. The sequence Alignment/Map format and SAMtools. Bioinformatics 2009, 25, 2078-2079. [CrossRef]

41. Wang, K.; Li, M.; Hakonarson, H. ANNOVAR: Functional annotation of genetic variants from high-throughput sequencing data. Nucleic Acids Res. 2010, 38, e164. [CrossRef]

42. Kim, D.; Langmead, B.; Salzberg, S.L. Hisat: A fast spliced aligner with low memory requirements. Nat. Methods 2015, 12, 357-360 [CrossRef] [PubMed]

43. Wang, S.-H.; Lim, J.-H.; Kim, S.-S.; Cho, S.-H.; Yoo, S.-C.; Koh, H.-J.; Sakuraba, Y.; Paek, N.-C. Mutation of SPOTTED LEAF3 (SPL3) impairs abscisic acid-responsive signaling and delays leaf senescence in rice. J. Exp. Bot. 2015, 66, 7045-7059. [CrossRef] [PubMed]

44. Zhang, X.; Henriques, R.; Lin, S.-S.; Niu, Q.-W.; Chua, N.-H. Agrobacterium-mediated transformation of Arabidopsis thaliana using the floral dip method. Nat. Protoc. 2006, 1, 641-646. [CrossRef] [PubMed] 